\title{
Nonthermal leptogenesis with almost degenerate superheavy neutrinos
}

\author{
Rouzbeh Allahverdi \\ Physik Department, TU München, James Frank Strasse, D-85748, Garching, Germany \\ Anupam Mazumdar \\ The Abdus Salam International Center for Theoretical Physics, I-34100 Trieste, Italy
}

(Received 5 September 2002; published 17 January 2003)

\begin{abstract}
We present a model with minimal assumptions for nonthermal leptogenesis with almost degenerate superheavy right-handed neutrinos in a supersymmetric setup. In this scenario the gauge singlet inflaton is directly coupled to the right-handed (s)neutrinos with a mass heavier than the inflaton mass. This helps to avoid potential problems which can naturally arise otherwise. The inflaton decays into standard model leptons and Higgs bosons via off-shell right-handed (s)neutrinos and reheats the Universe. The same channel is also responsible for generating the lepton asymmetry, thus requiring no stage of preheating in order to excite superheavy (s)neutrinos. The suppressed decay rate of the inflaton naturally leads to a sufficiently low reheat temperature, which in addition, prevents any wash out of the yielded asymmetry. We will particularly elaborate on important differences from leptogenesis with on-shell (s)neutrinos. It is shown that for nearly degenerate neutrinos a successful leptogenesis can be accommodated for a variety of inflationary models with a rather wide ranging inflationary scale.
\end{abstract}

DOI: 10.1103/PhysRevD.67.023509

PACS number(s): 98.80.Cq, 11.30.Fs, 12.60.Jv

\section{INTRODUCTION}

The consistency of the abundance of light elements synthesized during the big bang nucleosynthesis $(\mathrm{BBN})$ requires that the baryon asymmetry of the Universe (BAU), parametrized as $\eta_{\mathrm{B}}=\left(n_{\mathrm{B}}-n_{\overline{\mathrm{B}}}\right) / s$ with $s$ being the entropy density and $n_{B}$ the number density of the baryons, be in the range $(0.3-0.9) \times 10^{-10}[1]$. The asymmetry can be produced from the baryon symmetric universe provided three conditions are simultaneously met; $B$ and/or $L$ violation, $C$ and $C P$ violation, and departure from thermal equilibrium [2]. However, any produced asymmetry will be washed away by the standard model $(\mathrm{SM})(B+L)$-violating sphaleron transitions which are active from temperatures $10^{12} \mathrm{GeV}$ down to $100 \mathrm{GeV}$ [3], if $B-L=0$. Therefore, an asymmetry in $B$ $-L$ is generally sought which is subsequently reprocessed in a thermal bath via sphalerons in order to yield a net baryon asymmetry given by $B=a(B-L)$. Here, $a$ is a modeldependent parameter; in the case of the SM, $a=28 / 79$, while in the minimal supersymmetric standard model (MSSM), $a$ $=32 / 92$ [4].

An attractive mechanism for producing $B-L$ asymmetry is from the decay of heavy right-handed $(\mathrm{RH})$ Majorana neutrinos [6]. Since the RH neutrinos are SM singlets, a Majorana mass $M_{N}$, which violates lepton number, is compatible with all symmetries and hence can be arbitrarily large beyond the electroweak scale. This provides an elegant way for obtaining small masses $m_{\nu}$ for the light neutrinos via the seesaw mechanism such that $m_{\nu} \approx\left(m_{\mathrm{D}}^{2} / M_{N}\right)$ [5], where $m_{\mathrm{D}}$ is the Dirac mass obtained from the Higgs vacuum expectation value (VEV). Moreover, a lepton asymmetry can be generated from the interference between the tree-level and the one-loop diagrams in an out-of-equilibrium decay of the $\mathrm{RH}$ neutrinos, provided $C P$-violating phases exist in the neutrino Yukawa couplings. The lepton asymmetry thus obtained will be partially converted to the baryon asymmetry via sphaleron effects. This is the standard lore for producing lepton asymmetry commonly known as leptogenesis [6,7].

The present analyses of solar neutrino experiments favor the large mixing angle Mikheyev-Smirnov-Wolfenstein (MSW) solution with $\Delta m_{\nu, \text { solar }}^{2}=6.1 \times 10^{-5} \mathrm{eV}^{2}$ and $\tan ^{2} \theta_{12}=0.41 \quad[8]$, while $\Delta m_{\nu, a t m}^{2}=3.2 \times 10^{-3} \mathrm{eV}^{2}$ and $\sin ^{2}\left(2 \theta_{23}\right)=(0.83-1)$ provides the best fit to the atmospheric neutrino data [9].

In addition, cosmology [10] and neutrinoless double-beta decay experiments [11] provide an upper limit for the light neutrino masses. The masses and mixing angles which are required to explain solar and atmospheric neutrino data can be obtained in both scenarios with hierarchical, or quasidegenerate, neutrinos. Note that the hierarchical spectrum for heavy neutrinos strongly suggests a spectrum of light neutrinos which is hierarchical too unless there is a big conspiracy. On the other hand, a mild hierarchy of RH neutrino masses could be compatible with degenerate light neutrinos with a certain amount of fine-tuning. In the former case, one may consider a thermal leptogenesis scenario where heavy neutrinos come into equilibrium with the primordial thermal bath through Yukawa interactions. The decay of the lightest RH neutrino easily satisfies the out-of-equilibrium condition by virtue of having a sufficiently small Yukawa coupling [7]. In the model-independent analysis in Ref. [12], the authors have parametrized thermal leptogenesis by four parameters: the $C P$ asymmetry, the heavy $\mathrm{RH}$ neutrino mass, the effective light neutrino mass, and the quadratic mean of the light neutrino masses. The final result was that an acceptable lepton asymmetry can be generated with $T_{\mathrm{R}} \sim M_{1}$ $=\mathcal{O}\left(10^{10}\right) \mathrm{GeV}$ and $\sum_{i} m_{\nu, i}<\sqrt{3} \mathrm{eV}$.

However, the temperature required for thermal leptogenesis is marginally compatible with the maximum allowed one in supersymmetric theories, which is usually constrained by thermal gravitino production $[13,14]$. Gravitinos with a mass 
$\mathcal{O}(\mathrm{TeV})$ decay much after nucleosynthesis and their decay products can change the abundance of the light elements synthesized during BBN. For $100 \mathrm{GeV} \leqslant m_{3 / 2} \leqslant 1 \mathrm{TeV}$, a successful nucleosynthesis requires $n_{3 / 2} / s \leqslant\left(10^{-14}-10^{-12}\right)$, which translates into $T_{\mathrm{R}} \leqslant\left(10^{7}-10^{10}\right) \mathrm{GeV}[13,14] .{ }^{1}$ The possible ways for obtaining a naturally low reheat temperature include gravitationally suppressed decay of the inflaton in models of high scale inflation [22], low scale inflationary models [23-25], a brief period of late thermal inflation [26], or-a completely new paradigm "reheating through the surface evaporation" which works even for high scale inflationary models [27].

When the light neutrinos are almost degenerate $m_{\nu, 1}$ $\approx m_{\nu, 2} \approx m_{\nu, 3}$, which requires quasi-degenerate heavy neutrinos, the out-of-equilibrium condition in the thermal leptogenesis scenario cannot be satisfied in the minimal seesaw model [7]. More complicated models are required in this case [28]. On the other hand, if the mass splitting of the RH neutrinos becomes less than their decay widths, the perturbative calculations obviously break down. Then, the effect of finite decay widths of the RH neutrinos must be taken into account [29]. The careful treatment of Ref. [29] shows that a resonant enhancement of lepton asymmetry occurs in this case, while as expected, it vanishes in the limit of exactly degenerate neutrinos. This effect can be utilized to bring down the scale of heavy neutrino masses, and hence the leptogenesis scale [30].

However, for almost degenerate heavy neutrinos, i.e. where the mass splitting is larger than the decay width, one has to seek nonthermal leptogenesis (which works for the hierarchical neutrino masses as well) in the minimal models. In this scenario RH neutrinos are produced nonthermally from the inflaton decay. This can occur during reheating if the inflaton decays to the RH neutrinos, which are lighter than the inflaton, with a considerable branching ratio [31]. Heavy neutrinos can also be produced via preheating [32] (a stage of reheating where resonant production of massive and/or massless bosons and fermions takes place [33]) or tachyonic preheating [34], even if the mass of the boson and fermion exceeds that of the inflaton. All these are rather model-dependent and their main features can significantly vary from model to model. This is the prime reason why we do not pursue leptogenesis via preheating mechanism here.

In supersymmetric models one has the RH sneutrinos in addition. The sneutrinos are produced along with neutrinos

\footnotetext{
${ }^{1}$ Recently, nonthermal production of helicity $\pm 3 / 2[15]$ and helicity $\pm 1 / 2$ gravitinos $[16,17]$ from inflaton oscillations have been considered. For a single chiral multiplet the helicity $\pm 1 / 2$ gravitino is the superpartner of the inflaton known as the inflatino. The decay channels of inflatinos have been discussed in Ref. [18]. Also, it has been suggested [18], and explicitly shown [19], that in realistic models with two chiral multiplets the helicity $\pm 1 / 2$ gravitino production is not a problem, as long as the inflationary scale is sufficiently higher than the scale of supersymmetry breaking in the hidden sector and the two sectors are weakly coupled. Gravitinos can also be produced directly from the inflaton decay [20] and from the decay of the heavy stable neutral particles [21].
}

during reheating and with much higher abundances in preheating, thus serving as an additional source for leptogenesis [35]. Moreover, the RH sneutrinos can acquire a large VEV during inflation if their mass is less than the Hubble expansion rate during inflation $H_{I}$. Such a condensate starts oscillating once $H(t) \simeq M_{N}$, therefore automatically satisfying the out-of-equilibrium condition. The decay of the sneutrino condensate can then yield the desired lepton asymmetry in the same fashion as neutrino decay [36] or via the AffleckDine mechanism [37]. This last scenario has an additional advantage that it solves the fine-tuning problem in the $F$-term hybrid inflationary model in a very natural way [38].

The success of all these scenarios, but preheating and the Affleck-Dine oriented model, requires that the inflaton be heavier than the RH (s)neutrinos [in the hierarchical case inflaton only needs to be heavier than the lightest RH (s)neutrino]. Moreover, all the above scenarios are based upon the decay processes. An attractive proposal was recently made, where the lepton asymmetry in the visible sector is generated from the RH neutrino-mediated scattering of the SM Higgs bosons and leptons into a depleted hidden sector [39], rather than the decay of the on-shell heavy neutrinos.

In this paper we propose a simple supersymmetric model for nonthermal leptogenesis without any need of a preheating mechanism. In this model the inflaton is directly coupled to nearly degenerate RH (s)neutrinos which are heavier than the inflaton. Then the inflaton decays to the SM fields, via offshell RH (s)neutrinos, reheats the Universe and naturally leads to a sufficiently low reheat temperature. This same channel is also responsible for producing the lepton asymmetry.

In the next section we introduce our model and highlight several of its advantages. Then we turn to reheating and generation of the lepton asymmetry in this model and present our main results. In particular, we point out marked differences from leptogenesis with on-shell (s)neutrinos. Finally, we conclude the paper with a brief summary.

\section{MODEL}

We start by introducing our model in a supersymmetric set up. The relevant part of the superpotential is given by

$$
W \supset \frac{1}{2} m_{\phi} \Phi \Phi+\frac{1}{2} g \Phi \mathbf{N N}+h \mathbf{N H}_{u} \mathbf{L}+\frac{1}{2} M_{N} \mathbf{N N} .
$$

Here $\Phi, \mathbf{N}, \mathbf{L}$, and $\mathbf{H}$ stand for the inflaton, the RH neutrino, the lepton doublet, and the Higgs (which gives mass to the top quark) superfields, respectively. Also, $m_{\phi}$ and $M_{N}$ denote inflaton and RH (s)neutrino masses, respectively. ${ }^{2} \mathrm{We}$ assume that the inflaton is coupled to the RH (s)neutrinos via Yukawa coupling $g$, and $h$ denotes a typical neutrino Yukawa

\footnotetext{
${ }^{2}$ Actually, $m_{\phi}$ denotes the frequency of the inflaton oscillations around the global minimum of the potential. In models of chaotic inflation $m_{\phi} \simeq H_{I}$, while in new and hybrid inflationary models it is usually (much) larger than $H_{I}[40]$.
} 
coupling. For simplicity, we have omitted all indices in $h$ matrix and superfields, and work in the basis where the Majorana mass matrix is diagonal. Further simplifications can be made for almost degenerate RH (s)neutrinos where $M_{N}$ is essentially the same for all of them. It is also conceivable in this case that the inflaton is coupled with the same strength to three RH (s)neutrinos. This is particularly true when the inflaton has a nonzero VEV at the minimum which provides masses to the RH (s)neutrinos. We focus on superheavy RH (s)neutrinos, i.e. assuming that $M_{N} \gg m_{\phi}$.

Now let us discuss the merits why we seek RH (s)neutrinos heavier than the inflaton. If $M_{N}<m_{\phi}$, then one can easily produce on-shell (s)neutrinos from the inflaton decay, either perturbatively or via preheating. First consider (s)neutrino production in perturbative inflaton decay. A perturbative decay requires a small coupling to the (s)neutrinos. This is naturally achieved when the inflaton lies in a hidden sector which is only gravitationally coupled to the SM sector [22]. In this case the total decay rate of the inflaton is given by $\Gamma_{\mathrm{d}} \sim m_{\phi}^{3} / M_{\mathrm{P}}^{2}$, while the partial decay rate to (s)neutrinos is given by $\Gamma_{\phi \rightarrow N} \sim m_{\phi} M_{N}^{2} / M_{\mathrm{P}}^{2}$ [21], where $M_{\mathrm{P}}=2.4$ $\times 10^{18} \mathrm{GeV}$ is the reduced Planck mass. This results in a branching ratio $\simeq\left(M_{N} / m_{\phi}\right)^{2}$, which is too small if $M_{N}$ $\ll m_{\phi}$. Note that a successful leptogenesis requires an acceptable branching ratio given the entropy generation from reheating, thus implying that $M_{N}$ must not be much smaller than $m_{\phi}$.

Besides, a small coupling $g$, which is required to ensure a perturbative treatment, leads to another potential problem. The sneutrino field $\widetilde{N}$ can acquire a large VEV during inflation. Since $\widetilde{N}$ is directly coupled to the inflaton, it might even ruin the flatness of the inflaton potential. On a lighter note, $\langle\widetilde{N}\rangle$ remains non-vanishing after the end of inflation in any case and may contribute to isocurvature density perturbations [41]. This requires a delicate treatment of a coupled system which depends on the choice of a model. This is an issue which has been sidelined in most supersymmetric models of nonthermal leptogenesis, except Ref. [37].

If $g$ is sufficiently large, (s)neutrinos may be produced in a non-perturbative manner during the stage of (tachionic) preheating [32,34]. For the superpotential in Eq. (1), the necessary condition for preheating reads $g \phi_{0}>m_{\phi}$, where $\phi_{0}$ is the initial amplitude of the inflaton oscillations. This guarantees that $\widetilde{N}$ is heavier than the inflaton during inflation, and hence $\langle\widetilde{N}\rangle=0$ after the end of inflation, resulting in a simpler initial condition in the post-inflationary era. On the other hand, both RH neutrinos and sneutrinos can be produced via preheating (sneutrinos much more abundantly by virtue of obeying Bose statistics [33]). However, as mentioned earlier, this is rather model dependent. For example, if the inflaton has a VEV at the minimum, denoted as $v$, then it is hard to envisage an efficient production of (s)neutrinos through parametric resonance. The reason is that $M_{N}=g v$ and $\phi_{0} \simeq v$ in this case, which implies $g \phi_{0} \simeq M_{N}$. It is therefore evident that there will be no preheating of (s)neutrinos for $M_{N}$ $<m_{\phi}$. On the other hand, for $M_{N}>m_{\phi}$ preheating is possible only if $g \phi_{0} m_{\phi} \gg M_{N}^{2}[33,42]$. In particular, resonant creation rapidly ceases to be efficient for $M_{N}>10 m_{\phi}$ [42]. ${ }^{3}$ In the tachyonic preheating scenario, too, the produced (s)neutrinos usually have an abundance much less than the inflaton abundance when $M_{N} \gg m_{\phi}$ [34]. In conclusion, it is very difficult (if not impossible) to obtain the desired lepton asymmetry in a wide range of inflationary models, by solely relying on non-perturbative dynamics.

Now we count upon the advantages of our model. First of all, for $M_{N} \gg m_{\phi}$ the post-inflationary dynamics is simpler since $\langle\widetilde{N}\rangle=0$ at the end of inflation. The Universe is reheated through the inflaton decay to the Higgs boson and SM leptons via the off-shell RH (s)neutrino. The decay rate, as we will see shortly, is suppressed as $\left(m_{\phi} / M_{N}\right)^{4}$. This naturally leads to an acceptably low reheat temperature when $M_{N} \gg m_{\phi}$. Furthermore, the inflaton decay alone is responsible for the generation of the lepton asymmetry. This makes the model minimal since leptogenesis is now directly connected with reheating. Also, the washing out of the lepton asymmetry from thermal scattering of the SM leptons and Higgs boson is completely negligible since $T_{\mathrm{R}} \ll M_{N}$.

Our main focus will be on almost degenerate light neutrinos, which can be derived naturally from almost degenerate RH neutrinos. An example of such a model is presented in Ref. [44], where neutrino masses and mixing compatible with the solar and atmospheric neutrino solutions are derived in the framework of democratic mass matrix. There the neutrino Yukawa matrix $\mathbf{h}$ is almost diagonal in the same basis as the Majorana mass matrix. This makes sense since when both are proportional to the identity matrix the light neutrinos come out to be exactly degenerate. Then by perturbing around this pattern, we can obtain a nearly degenerate texture. In the calculations below, $M_{N}$ and $\Delta M_{N}$ denote the nearly equal diagonal elements of the Majorana mass matrix and their typical differences respectively. Also $h$ and $\delta h$ represent the nearly equal diagonal elements of the Yukawa matrix and their differences respectively, while $h^{\prime}$ stands for the typical non-diagonal elements. It is assumed that $\Delta M_{N}$ $<M_{N}$ and $h^{\prime}<\delta h<h$.

\section{REHEATING THE UNIVERSE}

The main decay mode of the inflaton is to a four-body final state consisting of two Higgs-boson/Higgsino-lepton/ slepton particles (and their $C P$ transforms). Since we have assumed $m_{\phi} \ll M_{N}$, it is essential to find those diagrams which are least suppressed by powers of $M_{N}$. These diagrams, shown in Fig. 1, which arise from the leading order terms in the effective superpotential after integrating out $\mathbf{N}$, are given by

\footnotetext{
${ }^{3}$ It has been shown in Ref. [42] that for a quadratic potential $V_{\phi}$ $\sim m_{\phi}^{2} \phi^{2}$, efficient resonant production of particles with a mass $M_{N}=10 m_{\phi}$ requires $g \phi_{0}>10^{4} m_{\phi}$. On the other hand, for a quartic potential $V_{\phi} \sim \lambda \phi^{4}$, preheating of these particles practically disappears. Preheating in the supersymmetric hybrid inflation model is also not efficient [43].
} 


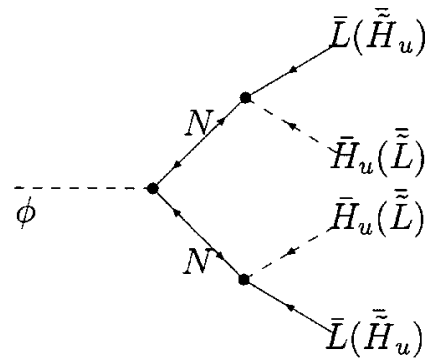

a) $\Delta L=-2$

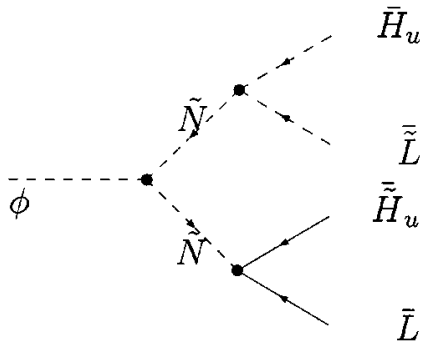

b) $\Delta L=-2$

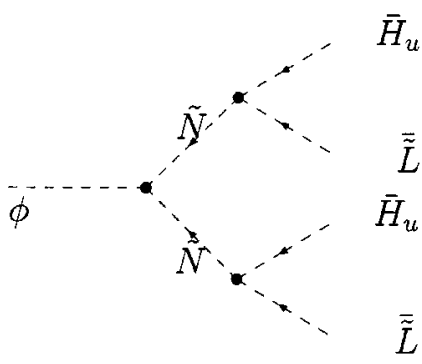

c) $\Delta L=-2$

FIG. 1. Diagrams together with their $C P$ transformed, for which $\Delta L=+2$, represent the inflaton decay into two Higgs-boson/ Higgsino-lepton/slepton pairs at leading order.

$$
W_{e f f} \supset \frac{1}{2} m_{\phi} \Phi^{2}+\frac{1}{2 M_{N}^{2}} g h^{2} \Phi\left(\mathbf{H}_{u} \mathbf{L}\right)\left(\mathbf{H}_{u} \mathbf{L}\right) .
$$

We should therefore choose that part of the $N$ propagator with a mass insertion, namely the part suppressed as $1 / M_{N}$ (the other part of the propagator is proportional to $m_{\phi} / M_{N}^{2}$ ). In the diagrams in Fig. 1 two opposite arrows on the $N$ propagator represent this dominant part. Note that $\widetilde{N}$ propagator is proportional to $\left(1 / M_{N}^{2}\right)$ to the leading order.

First, we evaluate the rate for inflaton decay without any specific assumption about Majorana masses and Yukawa couplings (except that $g$ is diagonal and universal, and $m_{\phi}$ $\left.\ll M_{N}\right)$. Generically, the trajectory of the inflaton motion is a line on a complex $\phi$ plane. We can therefore assume, without loss of generality, that only the real component of the inflaton has a VEV, thus treating the decaying inflatons as real fields. In addition, the SM particles are much lighter than the inflaton in the case under consideration (as will be confirmed by our results). Then the phase space factor for the four-body decay is readily found to be $\left[16 \times 96 \times(2 \pi)^{5}\right]^{-1}$. The inflaton coupling to a given final state consisting of $\bar{L}_{j}$ (or $\overline{\widetilde{L}}_{j}$ ) and $\bar{L}_{k}$ (or $\overline{\tilde{L}}_{k}$ ), plus two $\bar{H}_{u}$ (or $\overline{\widetilde{H}}_{u}$ ), is given by $\sum_{i} g h_{i j} h_{i k} / 2 M_{i}^{2}$. Here $j$ and $k$ stand for the lepton flavor. There is also a multiplicity factor for each final state which can be calculated easily.

Given all possible weak isospin assignments, with flavor indices fixed, there exist a total of nine final states. Seven of them which consist of two fermions and two scalars are (1)

$\bar{L}_{j}^{a} \bar{L}_{k}^{a} \bar{H}_{u}^{b} \bar{H}_{u}^{b}$,

(2) $\overline{\widetilde{L}}_{j}^{a} \overline{\widetilde{L}}_{k}^{a} \overline{\tilde{H}}_{u}^{b} \overline{\tilde{H}}_{u}^{b}$,

(3) $\bar{L}_{j}^{a} \bar{L}_{k}^{b} \bar{H}_{u}^{a} \bar{H}_{u}^{b}$,

$\overline{\widetilde{L}}_{j}^{a} \overline{\tilde{L}}_{k}^{b} \overline{\tilde{H}}_{u}^{a} \overline{\tilde{H}}_{u}^{b}$,

(5) $\bar{L}_{j}^{a} \overline{\tilde{L}}_{k}^{a} \bar{H}_{u}^{b} \overline{\tilde{H}}_{u}^{b}$,

(6) $\bar{L}_{j}^{a} \overline{\tilde{L}}_{k}^{b} \bar{H}_{u}^{a} \overline{\tilde{H}}_{u}^{b}$, and

$\bar{L}_{j}^{a} \overline{\tilde{L}}_{k}^{b} \bar{H}_{u}^{b} \overline{\tilde{H}}_{u}^{a}$. There are also two final states consisting of four scalars: (8) $\overline{\widetilde{L}}_{j}^{a} \overline{\widetilde{L}}_{k}^{a} \bar{H}_{u}^{b} \bar{H}_{u}^{b}$ and (9) $\overline{\tilde{L}}_{j}^{a} \overline{\widetilde{L}}_{k}^{b} \bar{H}_{u}^{a} \bar{H}_{u}^{b}$.

Note that at each vertex in diagram (a), the production of $\bar{L}^{a}$ (or $\overline{\widetilde{L}}^{a}$ ) is accompanied by that of $\bar{H}_{u}^{b}$ (or $\overline{\tilde{H}}_{u}^{b}$ ) and vice versa. In diagram (b), on the other hand, the production of $\bar{L}^{a}$ (or $\overline{\widetilde{L}}^{a}$ ) is accompanied by that of $\overline{\tilde{H}}_{u}^{b}$ (or $\bar{H}_{u}^{b}$ ) and vice versa. This implies that final states in (1)-(4) and (7) can arise from diagram (a), while (6) arises only from diagram (b). On the other hand, the final state in (5) can arise from both diagrams. Finally, (8),(9) arise only from diagram (c).

The rates for the inflaton decay to the final states in (1), (2) and (8) are the same and given by

$$
\begin{aligned}
\Gamma_{1} & =\Gamma_{2}=\Gamma_{8} \\
& \simeq \sum_{j \leqslant k}\left(2 \times\left[8-4 \delta_{j k}\right]\right) \times \frac{m_{\phi}^{5}}{16 \times 96 \times(2 \pi)^{5}}\left|\sum_{i} g \frac{h_{i j} h_{i k}}{2 M_{i}^{2}}\right|^{2} .
\end{aligned}
$$

The constraint $j \leqslant k$ is imposed in order to avoid double counting of the same final states. Note that the first number inside the parentheses comes from the summation over all isospin states, while the second one represents the overall factor from the superposition of different contributions for each isospin assignment.

Similarly, one can also evaluate the rates for the decay into other final states. The results are

$$
\Gamma_{3}=\Gamma_{4}=\Gamma_{9}=\frac{1}{2} \Gamma_{1},
$$

while

$$
\begin{aligned}
\Gamma_{6} & =\Gamma_{7}=\frac{1}{4} \Gamma_{5} \\
& \simeq \sum_{j, k}(2 \times 4) \times \frac{m_{\phi}^{5}}{16 \times 96 \times(2 \pi)^{5}}\left|\sum_{i} g \frac{h_{i j} h_{i k}}{2 M_{i}^{2}}\right|^{2} .
\end{aligned}
$$

The total decay rate of the inflaton will be 


$$
\Gamma_{\mathrm{d}}=\sum_{i=1}^{9} \Gamma_{i}
$$

Let us come back to the case with nearly degenerate neutrinos, where $\Delta M_{N}<M_{N}$ and $h^{\prime}<\delta h<h$. In this case each $N_{i}$ $\left(\widetilde{N}_{i}\right)$ is dominantly coupled to the $i$ th lepton doublet, and the coupling is $h$. In consequence, off-shell $N_{i}\left(\widetilde{N}_{i}\right)$ mainly contributes to the inflaton decay to the final states with $j=k$ $=i$. Then we can show that the decay rate will be given by

$$
\Gamma_{\mathrm{d}} \simeq \frac{21}{2^{14} \pi^{5}} g^{2} h^{4} \frac{m_{\phi}^{5}}{M_{N}^{4}} .
$$

The inflaton completely decays when $H \simeq \Gamma_{\mathrm{d}}$, where $H$ $\simeq\left(g_{*}^{1 / 2} T^{2} / M_{\mathrm{P}}\right)$ in a radiation-dominated universe [40], with $g_{*}$ being the effective number of relativistic degrees of freedom which is $\simeq 214$ in the MSSM. Assuming that thermal equilibrium is achieved when $H \simeq \Gamma_{\mathrm{d}}$ (which is justifiable; for a detailed discussion, see Refs. $[45,46])$, we obtain

$$
\frac{T_{\mathrm{R}}}{m_{\phi}} \simeq 10^{-7 / 2} \frac{g h^{2} m_{\phi}^{3 / 2} M_{\mathrm{P}}^{1 / 2}}{M_{N}^{2}} .
$$

Some comments are in order regarding our estimates of $\Gamma_{\mathrm{d}}$ and $T_{\mathrm{R}}$. One might think that the inflaton decaying into four scalars, the same as in diagram (b) except that $\overline{\widetilde{H}}_{u}$ and $\bar{L}$ are replaced with $H_{u}$ and $\widetilde{L}$, would occur at a rate only suppressed by two powers of $M_{N}$. However, this is not the case since this leading order contribution is canceled out by that from another diagram and the overall rate is actually proportional to $\left(m_{\phi}^{7} / M_{N}^{6}\right)$. This is just the manifestation that these diagrams do not arise from the effective superpotential individually. Also, there exists a two-body decay channel for the inflaton, into $\bar{H}_{u} H_{u}\left(\overline{\widetilde{H}}_{u} \widetilde{H}_{u}\right)$ or $\bar{L} L(\overline{\tilde{L}} \widetilde{L})$, at the one-loop level. It can easily be derived by choosing $\left(1 / M_{N}\right)$ and $\left(m_{\phi} / M_{N}^{2}\right)$ parts of $N$ propagators in diagram (a) and connecting the $\bar{H}_{u}(\overline{\tilde{L}})$, or $\bar{L}\left(\overline{\widetilde{H}}_{u}\right)$, lines. This channel has a much larger phase space factor $(8 \pi)^{-1}$, while the dependence on $g$ and $h$ remains the same as in Fig. 1. However, the two-body decay rate is $\propto\left(m_{\phi}^{7} / M_{N}^{6}\right)$. Thus, by taking the one-loop factor $(4 \pi)^{-2}$ into account and for $M_{N} \geqslant 10 m_{\phi}$, it will eventually be smaller than that in Eq. (7).

Finally, the inflaton can also decay into the SM fields via gravitational couplings with a decay rate $\Gamma_{\text {grav }}$ $\sim\left(v / M_{\mathrm{P}}\right)^{2}\left(m_{\phi}^{3} / M_{\mathrm{P}}^{2}\right)$, where $v$ denotes inflaton VEV at the global minimum of the potential [21]. Such a decay rate can however be neglected compared to the four-body decay provided $v \ll M_{\mathrm{P}}$.

\section{LEPTON ASYMMETRY}

In this section we evaluate the lepton asymmetry generated from the inflaton decay through diagrams in Fig. 1. First, we remind the reader that for the standard case where the decay of on-shell neutrinos yields the lepton symmetry, one has $\eta_{\mathrm{L}}=\Sigma_{i} \epsilon_{i}\left(n_{N_{i}} / s\right)$, where

$$
\epsilon_{i}=\sum_{i \neq j} \epsilon_{i j} ; \quad \epsilon_{i j}=-\frac{1}{8 \pi} \frac{1}{\left[\mathbf{h h}^{\dagger}\right]_{i i}} \operatorname{Im}\left(\left[\mathbf{h h}^{\dagger}\right]_{i j}\right)^{2} f\left(\frac{M_{j}^{2}}{M_{i}^{2}}\right)
$$

and [47]

$$
f(x)=\sqrt{x}\left(\frac{2}{x-1}+\ln \left[\frac{1+x}{x}\right]\right) .
$$

The first and second terms on the right-hand side of Eq. (10) correspond to the one-loop self-energy and vertex corrections, respectively. For hierarchical $N$, the following lower bound is found [48]:

$$
\left|\epsilon_{1}\right| \leqslant \frac{3}{8 \pi} \frac{M_{1} m_{3}}{\left\langle H_{0}\right\rangle^{2}},
$$

where $M_{1}$ and $m_{3}$ denote masses of the lightest heavy neutrino and the heaviest light neutrino respectively. Here $\left\langle H_{u}^{0}\right\rangle=174 \sin \beta \mathrm{GeV}$ is the VEV of $H_{u}$ in our vacuum, with $\tan \beta$ defined as the ratio of $\left\langle H_{u}^{0}\right\rangle$ and $\left\langle H_{d}^{0}\right\rangle$. On the other hand, $x \approx 1$ for almost degenerate RH neutrinos, and hence the self-energy contribution dominates. Then, it can be shown that (to the leading order) [44]

$$
\epsilon_{1} \simeq \epsilon_{2} \simeq \epsilon_{3} \simeq \frac{1}{4 \pi} \frac{h^{\prime 2}}{h^{2}} \frac{M_{N}}{\Delta M_{N}} \frac{M_{N}}{\left\langle H_{u}^{0}\right\rangle^{2}} \frac{\Delta m_{\nu, a t m}^{2}}{2 m_{\nu}} .
$$

Now we come back to our case, where the inflaton decay via off-shell $N(\widetilde{N})$ produces the lepton asymmetry. The net lepton asymmetry is generated from the interference between diagrams in Fig. 1 and the one-loop diagrams representing self-energy and vertex corrections to one of the $N(\widetilde{N})$ propagators. Diagrams with one-loop correction to both $N(\tilde{N})$ legs are of higher order and will be subdominant. There are major differences which arise in the analysis compared to the on-shell case, as we note in this following discussion. To demonstrate these differences explicitly, we focus on selfenergy and vertex corrections to diagram (a) of Fig. 1, shown in Fig. 2. Similar arguments will go through for the inflaton decay through diagrams (b) and (c) in Fig. 1.

Note that both $H_{u} L$ and $\widetilde{H}_{u} \widetilde{L}$ loops contribute to the selfenergy correction, while only one of them is relevant in the vertex correction for a given final state. Also, recall that only loops with on-shell particles make a contribution to the resultant asymmetry. Thus the self-energy and vertex loops involving $N_{l}$ actually represent $s$-channel and $t$-channel scattering of a Higgs-lepton or Higgsino-slepton pair via offshell $N_{l}$, respectively. The center-of-mass energy available in these processes is at most equal to the inflaton mass. In consequence, the self-energy correction is simply twice as large as the vertex correction for $m_{\phi} \ll M_{N} \cdot{ }^{4}$ It can also be

\footnotetext{
${ }^{4}$ This is similar to the $x \gg 1$ limit for the standard case in Eq. (10).
} 


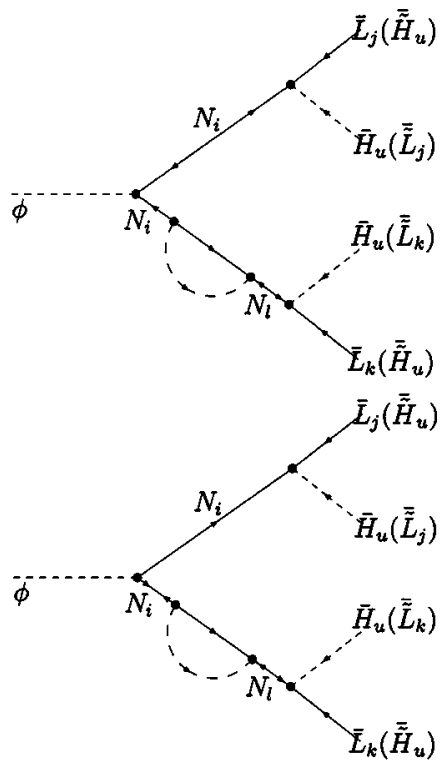

a)
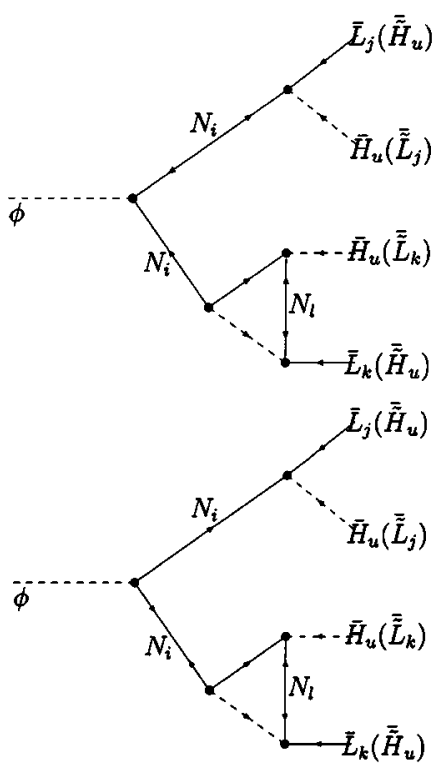

b)

FIG. 2. Diagrams representing one-loop (a) self-energy, and (b) vertex corrections to the decay channel shown in Fig. 1(a). The interference between these and the tree-level diagram results in a net lepton asymmetry.

shown that only the mass insertion part of the $N_{l}$ propagator contributes to the generated asymmetry from the self-energy correction of $N_{i}$. The diagram with mass insertion in the $N_{i}$ propagator will be irrelevant, exactly like the standard case [47].

An important difference arises in comparison with the standard case that there the center-of-mass energy in the twobody decay of $N_{i}$ is simply determined by $M_{i}$, while here the energy flowing in the $N_{i}$ leg is $0<E<m_{\phi}$. In the $m_{\phi}$ $\ll M_{N}$ limit, the $N_{i}$ propagator is $E / M_{i}^{2}$, while the $N_{l}$ propa- gator will simply be $1 / M_{l}$; see the diagrams in Fig. 2 . For a given final state with definite momenta the one-loop diagram is suppressed as $E^{2} / M_{i} M_{l}$ with respect to the tree-level one. Upon performing phase space integration over a four-body final state we find the suppression will be $m_{\phi}^{2} / M_{i} M_{l}$ times some numerical factor $\sim \mathcal{O}(1)$. For simplicity, we take the average energy in the $N_{i}$ legs to be $m_{\phi} / 2$, and hence the suppression comes as $\simeq m_{\phi}^{2} / 4 M_{i} M_{l}$. This approximation is adequate for our purposes in the $m_{\phi} \ll M_{N}$ limit, and any difference from the exact result will be numerically irrelevant. The reason is that the main contribution to the phase space integral comes from the bulk of the available phase space, while the contribution of the parts in which the energy of some decay products is $\ll m_{\phi}$, including parts with $E$ $\approx 0$ or $E \approx m_{\phi}$, is suppressed. The situation will be more complicated when $m_{\phi}$ and $M_{N}$ are not very different, since the energy and momentum carried by $N$ legs are comparable to $M_{N}$. In such a case the $N_{i}$ and $N_{l}$ propagators can strongly depend on the phase space distribution of the decay products and the above approximation may not be sufficient.

Now let us find the asymmetry parameter in the inflaton decay. First consider the diagrams in Figs. 1(a) and 2. For a given final state the tree-level and interference terms naturally have the same multiplicity factor. As explained earlier the self-energy correction is twice as large as the vertex correction, and also, the average energy carried by each of the $N$ legs can be approximately taken to be $m_{\phi} / 2$. The contributions from both diagrams in Figs. 2(a) and 2(b) are equal, and hence the asymmetry receives an extra factor of $3 m_{\phi}^{2} / 2 M_{N}^{2}$, in addition to the $(1 / 8 \pi)$ prefactor in Eq. (9). Note that the one-loop correction can come from each of the two $N$ legs, which is equivalent to exchanging $j$ with $k$. The situation will be similar for the asymmetry in the inflaton decay through diagrams (b) and (c) in Fig. 1. Thus, after summing over all possible final states, we obtain

$$
\epsilon \simeq-\frac{3}{8 \pi} \times \frac{\sum_{i, n, l} \frac{\operatorname{Im}\left[\left(\mathbf{h} \mathbf{h}^{\dagger}\right)_{n i}\left(\mathbf{h h}^{\dagger}\right)_{n l}\left(\mathbf{h h}^{\dagger}\right)_{i l}\right] m_{\phi}^{2}}{M_{i}^{3} M_{n}^{2} M_{l}}}{\sum_{i, n} \frac{\left(\left[\mathbf{h h}^{\dagger}\right]_{i n}\right)^{2}}{M_{i}^{2} M_{n}^{2}}}
$$

which is functionally very different from the standard case in Eq. (9).

We now come back to the case with nearly degenerate neutrinos. Now, the denominator of the second term on the right-hand side of Eq. (13) is $\simeq 3 h^{4} / M_{N}^{4}$. In this case the Yukawa matrix $\mathbf{h}$ is almost diagonal, and so is the matrix $\mathbf{h h}^{\dagger}$. The numerator receives the main contribution from the terms with $i=n$ and $i=l,{ }^{5}$ and can be written as

$$
\sum_{i \neq l} h^{2} \operatorname{Im}\left(\left[\mathbf{h h}^{\dagger}\right]_{i l}\right)^{2} m_{\phi}^{2}\left(\frac{1}{M_{i}^{5} M_{l}}-\frac{1}{M_{i}^{4} M_{l}^{2}}\right) .
$$

\footnotetext{
${ }^{5}$ Terms with $n=l$ and $i=n=l$ are real, and hence do not contribute to the asymmetry.
} 
This can further be simplified to $12 h^{3} \delta h h^{\prime 2}\left(m_{\phi}^{2} \Delta M_{N} / M_{N}^{7}\right)$, assuming that only the non-diagonal elements contain $C P$-violating phases. Since the lepton number is violated by two units in the inflaton decay, we finally have

$$
\frac{n_{L}}{n_{\phi}} \simeq \frac{3}{\pi} \frac{\delta h h^{\prime 2}}{h} \frac{\Delta M_{N}}{M_{N}}\left(\frac{m_{\phi}}{M_{N}}\right)^{2} .
$$

An important observation is that here the final asymmetry is proportional to $\Delta M_{N}$, contrary to the on-shell case in Eq. (12). Therefore the generated asymmetry actually decreases as the RH (s)neutrinos become more degenerate. This is not difficult to understand as the available energy in the inflaton decay $m_{\phi}$ is far below the mass of the RH (s)neutrinos $M_{N}$, independently of how degenerate the latter ones are.

The total asymmetry in the baryons (after taking into account of sphaleron effects) can be expressed as

$$
\eta_{\mathrm{B}}=\left(\frac{n_{B}}{n_{\phi}}\right)\left(\frac{n_{\phi}}{s}\right) \simeq \frac{1}{\pi} \frac{\delta h h^{\prime 2}}{h^{3}} \frac{\Delta M_{N}}{M_{N}}\left(\frac{M_{N} m_{\nu}}{\left\langle H_{u}^{0}\right\rangle^{2}}\right)\left(\frac{m_{\phi}}{M_{N}}\right)^{2}\left(\frac{T_{\mathrm{R}}}{m_{\phi}}\right),
$$

where $s=\left(2 \pi^{2} / 45\right) g_{*} T_{\mathrm{R}}^{3}$. Here $n_{\phi} / s$ denotes the dilution from reheating. By using Eq. (8) and the relationship $m_{\nu}$ $\simeq\left(h^{2}\left\langle H_{u}^{0}\right\rangle^{2} / M_{N}\right)$, we eventually obtain

$$
\eta_{\mathrm{B}} \simeq 4.10^{-49 / 2} g \frac{\delta h h^{\prime 2}}{h^{3}} \frac{\Delta M_{N}}{M_{N}} \frac{m_{\phi}^{7 / 2} M_{\mathrm{P}}^{1 / 2}}{M_{N}^{2}\left\langle H_{u}^{0}\right\rangle^{4}}(1 \mathrm{GeV})^{2},
$$

where we have taken $m_{\nu} \approx 0.1 \mathrm{eV}$. We also assume $\left\langle H_{u}^{0}\right\rangle$ $=174 \mathrm{GeV}$ in below. Moreover, for $\Delta M_{N} \simeq M_{N}$ and as long as $h^{\prime}<\delta h$, it is sufficient to have $\delta h / h \approx \Delta M_{N} / 2 M_{N}$ in order to obtain degenerate light neutrino masses. Therefore, we may further simplify Eq. (17) to find

$$
\eta_{\mathrm{B}} \simeq 2.10^{-49 / 2} g \frac{h^{\prime 2}}{h^{2}}\left(\frac{\Delta M_{N}}{M_{N}}\right)^{2} \frac{m_{\phi}^{7 / 2} M_{\mathrm{P}}^{1 / 2}}{M_{N}^{2}\left\langle H_{u}^{0}\right\rangle^{4}} \quad(1 \mathrm{GeV})^{2} .
$$

Let us now present some numerical examples for nearly degenerate superheavy RH (s)neutrinos, i.e. $M_{N} \geqslant 10 m_{\phi}$ and $\Delta M_{N} \simeq M_{N}$. With $M_{N}=10 m_{\phi}$ and $10^{-1} \leqslant h^{\prime} / h \leqslant 1$, the desired baryon asymmetry can be obtained for the range of parameters $10^{-3} \leqslant g \leqslant 1$ and $10^{11} \mathrm{GeV} \leqslant m_{\phi} \leqslant 10^{13} \mathrm{GeV}$, which result in $10^{6} \mathrm{GeV} \leqslant T_{\mathrm{R}} \leqslant 10^{8} \mathrm{GeV}$. With $M_{N}$ $=100 m_{\phi}$, and $10^{-1} \leqslant h^{\prime} / h \leqslant 1$ as before, an acceptable asymmetry is yielded for $g=1$ and $m_{\phi} \simeq 10^{12}-10^{13} \mathrm{GeV}$, with $10^{7} \mathrm{GeV} \leqslant T_{\mathrm{R}} \leqslant 10^{9} \mathrm{GeV}$.

The merits of our model are already evident from these numbers. First of all, the reheat temperature is low (more than) enough to avoid the gravitino problem. Moreover, $T_{\mathrm{R}}$ $\ll M_{N}$ guarantees that lepton number violating scattering of the SM particles is completely negligible, especially keeping in mind that in the MSSM there are a large number of scattering processes which can considerably attenuate the obtained asymmetry if the reheat temperature $T_{\mathrm{R}}$ is close to $M_{N}$ [7]. In our case, obtaining a sufficiently low reheat tempera- ture is more than welcome in this regard. Also, the robustness of the inflaton mass $m_{\phi}$ lies in a range compatible with both high and intermediate scale inflationary models, though slightly favoring high scale models, thus making the scenario more flexible.

We shall re-emphasize the marked difference from leptogenesis with on-shell (s)neutrinos, namely suppression of the yielded asymmetry as $\Delta M_{N} / M_{N}$ decreases. This implies that our scenario works well for nearly degenerate neutrinos (and perhaps even better in the hierarchical case), while producing too little asymmetry for highly degenerate ones. Note that no resonant enhancement of the lepton asymmetry of the type discussed in Ref. [29] will occur. However, we can expect a qualitatively similar effect if (at least) one of the RH sneutrinos is almost degenerate with the inflaton.

We would like to make a final comment before closing this section. A small number of on-shell (s)neutrinos might also have been produced non-perturbatively from an inefficient preheating and hence contribute to the resultant asymmetry through their decay. The asymmetry yielded in the decay of on-shell particles, denoted as $\eta_{\mathrm{B}}^{o n}$, will be

$$
\eta_{\mathrm{B}}^{o n} \simeq \frac{M_{N}^{4}}{3 \Delta M_{N}^{2} m_{\phi}^{2}}\left(\frac{n_{\tilde{N}}+n_{N}}{n_{\phi}}\right) \eta_{\mathrm{B}} .
$$

Note that the asymmetry parameter for on-shell (s)neutrinos is dominated by the self-energy correction, given in Eq. (10), and hence $\eta_{\mathrm{B}}^{o n}$ does not contain the suppression factor $\left(m_{\phi} / 2 M_{N}\right)^{2}$. On the other hand, a factor of 4 will be lost, relative to the off-shell case, since the one-particle decay of on-shell $\widetilde{N}$ and $N$ violates the lepton number by one unit. Thus, with $\Delta M_{N} \simeq M_{N}$, the possible contribution from onshell (s)neutrinos can be neglected, provided $\left(n_{\tilde{N}}+n_{N}\right)$ $<\left(3 m_{\phi}^{2} / M_{N}^{2}\right) n_{\phi}$. For the range of parameters considered above this is generically the case.

\section{CONCLUSION}

In this paper we have provided a simple example for nonthermal leptogenesis with nearly degenerate superheavy $\mathrm{RH}$ neutrinos in a supersymmetric setup. We assumed that the inflaton is lighter than the RH (s)neutrinos, thus naturally avoiding some potential problems which can naturally arise. The inflaton decay via off-shell (s)neutrinos reheats the Universe and the model is minimal in a sense that the same channel is also responsible for generating the lepton asymmetry. As usual, the asymmetry arises from the interference between the tree-level and the one-loop diagrams representing self-energy and vertex corrections of (s)neutrinos, although off-shell in our case, provided neutrino Yukawa couplings contain $C P$-violating phases. However, there are important differences from leptogenesis with on-shell (s)neutrinos, which we have pronounced here. The selfenergy and vertex corrections are now of the same order regardless of the degree of degeneracy. Most notably, the asymmetry parameter is found to be linearly proportional (rather than inversely in the on-shell case) to the mass difference of the RH (s)neutrinos. This results in a lepton asym- 
metry which gets smaller as the RH (s)neutrinos become more degenerate.

Finally, we briefly emphasize the remarkable advantages of this model. First of all leptogenesis can be accommodated rather simply without relying on non-perturbative production of RH (s)neutrinos. It is particularly attractive that the desired baryon asymmetry can be directly generated in the final stage of reheating which is perturbative, regardless of any model-dependent effects which might have resulted in a first stage of non-perturbative reheating. Second, the suppressed decay of the inflaton naturally leads to an acceptably low reheat temperature, which is compatible with the gravitino bound and also prevents any washout of the yielded asymmetry. Also, with nearly degenerate (s)neutrinos, the desired lepton asymmetry can be generated for a range of inflaton mass accessible in large and intermediate scale models of inflation.
Qualitatively, we expect that this scenario also works (even better) in the case of hierarchical RH (s)neutrinos. However, a more careful study should be performed in order to compare the quantitative results with those obtained here. It will also be interesting to study the possible enhancement of the lepton asymmetry when the inflaton is almost degenerate with some of the RH sneutrinos.

\section{ACKNOWLEDGMENTS}

We wish to thank Z. Berezhiani, W. Büchmuller, M. Drees, B. Dutta, and A. Perez-Lorenzana for useful discussions. The work of R.A. was supported by "Sonderforschungsbereich 375 für Astro-Teilchenphysik" der Deutschen Forschungsgemeinschaft. A.M. acknowledges the support of The Early Universe Network HPRN-CT-2000-00152.
[1] For a recent review, see K.A. Olive, hep-ph/0202486.

[2] A.D. Sakharov, JETP Lett. 91, 24 (1967).

[3] V. Kuzmin, V.A. Rubakov, and M.E. Shaposhnikov, Phys. Lett. 155B, 36 (1985).

[4] S.Yu. Khlebnikov and M.E. Shaposhnikov, Nucl. Phys. B308, 885 (1985).

[5] M. Gell-Mann, P. Ramond, and R. Slansky, in Supergravity, edited by P. van Nieuwenhuizen and D. Z. Freedman (NorthHolland, Amsterdam, 1979); T. Yanagida, in Proceedings of the Workshop on Unified Theory and Baryon number in the Universe, Tsukuba, Japan, 1979, edited by O. Sawada and A. Sugamoto (KEK Report No. 79-18, Tsukuba, 1979); R.N. Mohapatra and G. Senjanovic, Phys. Rev. Lett. 44, 912 (1980).

[6] M. Fukugita and T. Yanagida, Phys. Lett. B 174, 45 (1986).

[7] M. Plümacher, Z. Phys. C 74, 549 (1997); Nucl. Phys. B530, 207 (1998); W. Büchmuller and M. Plümacher, Phys. Rep. 320, 329 (1999); Int. J. Mod. Phys. A 15, 5047 (2000).

[8] P. C. de Holanda and A. Yu. Smirnov, Phys. Rev. D 66, 113005 (2002).

[9] G.L. Fogli, E. Lisi, A. Marrone, D. Montanino, and A. Palazzo, Nucl. Phys. B (Proc. Suppl.) 110, 268 (2002).

[10] A.D. Dolgov, Phys. Rep. 370, 333 (2002); G.G. Raffelt, hep-ph/0208024.

[11] H.V. Kalpdor-Kleingrothaus, A. Dietz, H.L. Harney, and I.V. Krivosheina, Mod. Phys. Lett. A 16, 2409 (2002).

[12] W. Büchmuller, P. Di Bari, and M. Plümacher, Nucl. Phys. B643, 637 (2002).

[13] J. Ellis, J.E. Kim, and D.V. Nanopoulos, Phys. Lett. 145B, 181 (1984); J. Ellis, D.V. Nanopoulos, K.A. Olive, and S.-J. Rey, Astropart. Phys. 4, 371 (1996); for a recent calculation, see M. Boltz, A. Brandenburg, and W. Büchmuller, Nucl. Phys. B606, 518 (2001).

[14] For a review, see S. Sarkar, Rep. Prog. Phys. 59, 1493 (1996).

[15] A.L. Maroto and A. Mazumdar, Phys. Rev. Lett. 84, 1655 (2000).

[16] R. Kallosh, L. Kofman, A.D. Linde, and A. Von Proeyen, Phys. Rev. D 61, 103503 (2000); G.F. Giudice, I.I. Tkachev, and A. Riotto, J. High Energy Phys. 08, 009 (1999).
[17] M. Bastero-Gil and A. Mazumdar, Phys. Rev. D 62, 083510 (2000).

[18] R. Allahverdi, M. Bastero-Gil, and A. Mazumdar, Phys. Rev. D 64, 023516 (2001).

[19] H.P. Nilles, M. Peloso, and L. Sorbo, Phys. Rev. Lett. 87, 051302 (2001); J. High Energy Phys. 04, 004 (2001).

[20] H.P. Nilles, K.A. Olive, and M. Peloso, Phys. Lett. B 522, 304 (2001).

[21] R. Allahverdi, K. Enqvist, and A. Mazumdar, Phys. Rev. D 65, 103519 (2002).

[22] G.G. Ross and S. Sarkar, Nucl. Phys. B461, 597 (1996).

[23] L. Randall, M. Soljacic, and A.H. Guth, Nucl. Phys. B472, 377 (1996).

[24] A. Mazumdar, Phys. Lett. B 469, 55 (1999).

[25] G. Germàn, G.G. Ross, and S. Sarkar, Nucl. Phys. B608, 423 (2001).

[26] D.H. Lyth and E.D. Stewart, Phys. Rev. Lett. 75, 201 (1995); Phys. Rev. D 53, 1784 (1996).

[27] K. Enqvist, S. Kasuya, and A. Mazumdar, Phys. Rev. Lett. 89, 091301 (2002); Phys. Rev. D 66, 043505 (2002).

[28] E. Ma and U. Sarkar, Phys. Rev. Lett. 80, 5716 (1998); E.J. Chun and S.K. Kang, Phys. Rev. D 63, 097902 (2001).

[29] A. Pilaftsis, Int. J. Mod. Phys. A 14, 1811 (1999).

[30] T. Hambye, Nucl. Phys. B633, 171 (2002).

[31] G. Lazarides and Q. Shafi, Phys. Lett. B 258, 305 (1991); K. Kumekawa, T. Moroi, and T. Yanagida, Prog. Theor. Phys. 92, 437 (1994); G. Lazarides, in Symmetries in Intermediate and High Energy Physics, Springer Tracts in Modern Physics Vol. 163, edited by A. Faessler, T.S. Kosmas, and G.K. Leontaris (Springer-Verlag, Berlin, 2000), p. 227; T. Asaka, K. Hamaguchi, M. Kawasaki, and T. Yanagida, Phys. Lett. B 464, 12 (1999); Phys. Rev. D 61, 083512 (2000).

[32] G.F. Giudice, M. Peloso, A. Riotto, and I.I. Tkachev, J. High Energy Phys. 08, 014 (1999).

[33] J. Traschen and R. Brandenberger, Phys. Rev. D 42, 2491 (1990); L. Kofman, A.D. Linde, and A.A. Starobinsky, Phys. Rev. Lett. 73, 3195 (1994); Phys. Rev. D 56, 3258 (1997).

[34] J. Garcia-Bellido and E. Ruiz Morales, Phys. Lett. B 536, 193 (2002). 
[35] B.A. Campbell, S. Davidson and K.A. Olive, Nucl. Phys. B399, 111 (1993).

[36] H. Murayama and T. Yanagida, Phys. Lett. B 322, 349 (1994); K. Hamaguchi, H. Murayama, and T. Yanagida, Phys. Rev. D 65, 043512 (2002).

[37] Z. Berezhiani, A. Mazumdar, and A.P. Lorenzana, Phys. Lett. B 518, 282 (2001).

[38] Z. Berezhiani, D. Comelli, and N. Tetradis, Phys. Lett. B 431, 286 (1998).

[39] L. Bento and Z. Berezhiani, Phys. Rev. Lett. 87, 231304 (2001)

[40] A. D. Linde, Particle Physics and Inflationary Cosmology (Harwood, New York, 1990).

[41] A.R. Liddle and A. Mazumdar, Phys. Rev. D 61, 123507 (2000).
[42] S.Yu. Khlebnikov and I.I. Tkachev, Phys. Lett. B 390, 80 (1997).

[43] D. Cormier, K. Heitmann, and A. Mazumdar, Phys. Rev. D 65, 083521 (2002).

[44] M. Fujii, K. Hamaguchi, and T. Yanagida, Phys. Rev. D 65, 115012 (2002).

[45] S. Davidson and S. Sarkar, J. High Energy Phys. 11, 012 (2000).

[46] R. Allahverdi and M. Drees, Phys. Rev. D 66, 063513 (2002).

[47] M. Flanz, E.A. Paschos, and U. Sarkar, Phys. Lett. B 345, 248 (1995); L. Covi, E. Roulet, and F. Vissani, ibid. 384, 169 (1996); W. Büchmuller and M. Plümacher, ibid. 431, 354 (1998).

[48] S. Davidson and A. Ibarra, Phys. Lett. B 535, 25 (2002). 